\title{
A MONOLITHIC POLYIMIDE NOZZLE ARRAY FOR INKJET PRINTING
}

\author{
Jingkuang Chen, Wenhan Juan*, Joel Kubby, and Biay-Cheng Hseih \\ Xerox Wilson Research Center \\ 800 Phillips Road/Bldg. 114-41D, Webster, NY 14580 \\ *Center for Integrated Sensors and Circuits, The University of Michigan \\ Ann Arbor, MI 48109-2122
}

\begin{abstract}
A monolithic polyimide nozzle array for thermal inkjet printing has been developed. This print head was realized by molding polyimide nozzles on top of a silicon wafer with a micro heater array. On this print head, closely spaced nozzles and $22 \mu \mathrm{m}$ high micro cavities/channels are formed using a direct photolithographic process on a photosensitive polyimide and a sacrificial etch on a thick photoresist using a low temperature $\left(<300^{\circ} \mathrm{C}\right)$ process. The micro cavities under each nozzle are separated from each other by polyimide columns which in this design have a minimum width of $6 \mu \mathrm{m}$. All the micro cavities connect to a common ink reservoir which is formed during the same sacrificial etch used to build the ink cavities. Ink is supplied to the reservoir through a hole in the silicon substrate. Nozzle arrays with densities ranging from 300 dots per inch (dpi) to 800 dpi have been designed and fabricated. Different geometrical configurations of the ink cavity-reservoir connection have also been designed and tested for optimizing the ink supply efficiency.
\end{abstract}

\section{INTRODUCTION}

The rapid advance of inkjet printing [1] technology has changed the nature of the consumer printer market as well as brought significant impact on many areas which are related to images/text production. Inkjet printers produce images/texts by ejecting ink drops onto paper/medium through a uniformly spaced nozzle array. Because of its low cost, lightweight, compactness, and capability of producing high quality color images/text, inkjet printing has become the technology of choice for personal computers over the past decade. With a continuous R\&D effort which improve the the image quality of inkjet printing, the inkjet printers market is expanding from low-end marking to high-end printing and even to areas that traditionally did not belong to direct marking, for example, printing of photograph and color inkjet copying.

The key factors that support the success of the inkjet market include the high image quality and the low device cost. Over the past twenty years, much effort has been made to improve the image quality of inkjet printing. For example, different nozzle structures have been designed for increasing the printing resolution, different actuators have been explored to enhance the gray scale and the energy efficiency, and different inks have been developed for producing more vivid images and for reducing ink smearing. The advance of inkjet printing is remarkable: twenty years ago, the images it produced were not much better than that of a dot matrix printer, however, today an inkjet printer can print images comparable to that of a laser printer.
Printer cost is the other important factor that drives the rapid growth of the inkjet market. With its comparatively simple structure, the cost of an inkjet system is significantly lower than other printing technologies that can produce high quality color images. The major component of an inkjet system is the printhead. It is composed of a nozzle array, an ink reservoir that supplies ink to the nozzles, micro actuators for ejecting an ink droplet, and control circuitry. Currently, many commercial inkjet nozzle heads are realized using bonding technology to assemble the fluidic and microelectronic components together. Each component is built separately using different processes. These approaches typically require relatively complicated fabrication processes and therefore increase product cost.

In this paper, a monolithic approach is used to fabricate an inkjet nozzle. This print head was realized by molding polyimide nozzles on top of a silicon wafer with a micro heater array using a low temperature $\left(<300^{\circ} \mathrm{C}\right)$ process. The process was designed to be IC compatible such that it can be mass produced in a foundry. Compared with metal micro structures realized using electroplating [2], micro structures made of polyimide introduce less thermal crosstalk and provide more process flexibility for inkjet applications. Polyimide is mechanically strong and has been widely studied in various micro sensor/actuator applications [3]. Polyimide is stable in inks which are free of the $\mathrm{OH}$ ion, and has been broadly used in inkjet nozzle head fabrication either as the passivation layer for protecting circuitry or as a part of the nozzle structure. Polyimide can be shaped to various micro structures, either through a direct photolithographic process or a dry etch. While most photoresists dissolve in acetone, polyimide does not and therefore photoresist can be used as a sacrificial layer for undercutting a polyimide microstructure. The construction of polyimide micro structures is a low temperature process and compatible with MOS circuitry integration as well as some wet etch processes for surface and/or bulk micromachining. Because of these merits, polyimide was chosen to fabricate the nozzle array. The design, fabrication and testing of such a monolithic polyimide nozzle head will be described in the following sections.

\section{PRINTHEAD DESIGN}

Figure 1 shows a perspective view of this polyimide nozzle head. The substrate of this printhead is a (100) silicon wafer, which supports the heater array and provides via holes for ink supply. On top of the silicon substrate, a polyimide manifold which includes nozzles, ink cavities, and part of the front-end ink reservoir is monolithically molded on the silicon substrate. The polyimide nozzles, which sit on $22 \mu \mathrm{m}$ high micro cavities, are used 
for guiding ink ejection. These micro cavities are separated from each other by polyimide columns which in this design have a minimum width of $6 \mu \mathrm{m}$ and a height of approximately $22 \mu \mathrm{m}$. Under each micro cavity is a thin-film heater, which is used for heating ink to generate the actuation force to expel an ink droplet. All the micro cavities connect to a common ink reservoir which is formed during the same sacrificial etch used to build the ink cavities. Ink is supplied to the reservoir through a hole etched from the back side of the silicon substrate. Nozzle arrays with densities ranging from 300 dots per inch (dpi) to $800 \mathrm{dpi}$ have been designed and fabricated.

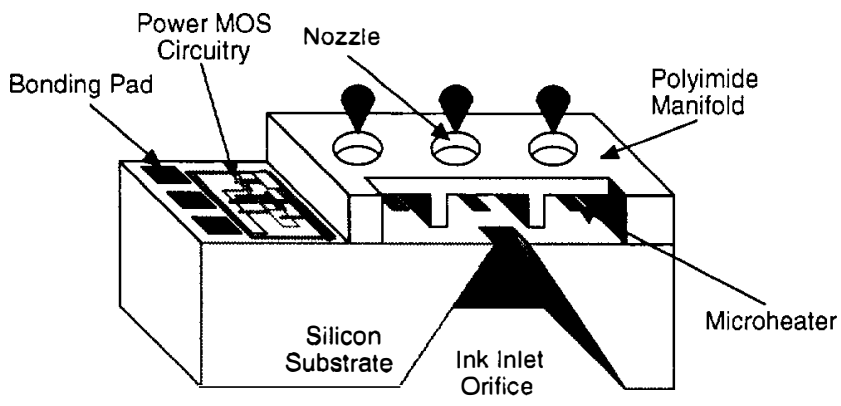

Figure 1: Perspective view of a monolithic inkjet printhead which is composed of a polyimide manifold on top of a silicon substrate.

In this design, the thin film resistors are used as heaters for drop ejection as well as temperature sensors for monitoring the heater temperature variation during the ejection process. The temperature resolution of such thin film resistors can be as high as $0.1^{\circ} \mathrm{C}$ if appropriate material and doping concentration are used [4].

The ink refilling process is one of the major factors that limits the printing speed of an inkjet system. After a drop of ink is ejected out of a nozzle, the nozzle cannot be fired again until after it is refilled from the reservoir. Typically, the major driving force for the refilling process is capillary action. For a circular tube of internal radius $r$, the movement of liquid inside this tube due to capillary action can be described by [5]

$$
\frac{d(\rho A L V)}{d t}=2 \pi r \sigma(\cos \Theta)-f
$$

Where $\rho$ is the density of the liquid, $A$ is the internal cross sectional area of the tube, $\mathrm{L}$ is the length of the tube that is filled with the liquid, $\mathrm{V}$ is the velocity of the fluid driven by the capillary force, $\sigma$ is the surface tension force, $\Theta$ is the contact angle of the liquid to the internal wall of the tube, and $f$ is the friction force. The behavior of fluid in a pipe with a non-circular cross section can be described by a similar formula in which the term $2 \pi \mathrm{r}$ is replaced by the internal perimeter length of the tube. With a given boundary condition this equation can be solved using a numerical method and the transient behavior of the ink in the chamber can be predicted.

In order to reduce the ink refill time, either the crosssectional area or the number of supply channels needs to be increased. However, these approaches generally degrade the ejector efficiency, which is defined as the ratio of the volume of ink expelled out of the nozzle to the total volume change of the cavity [6]. This is because in this case, a significant portion of the actuation momentum is used to push the ink back into the ink supply. In order to achieve an optimal design, different geometrical configurations of the ink cavity-reservoir connection have been designed and verified using numerical analysis. It was found that it is possible to achieve increased refilling speed with an acceptable ejection efficiency by using some multiple channelsupply arrangements.

\section{DEVICE FABRICATION}

The fabrication of this inkjet nozzle head started with the formation of a thin film micro heater array on a (100) silicon wafer. On top of the heater, dielectrics composed of stress balanced oxide/nitride/oxide triple layers were deposited using Low Pressure Chemical Vapor Deposition (LPCVD). Via holes for ink supply were then etched through the wafer using a wet etch, as shown in Figure 2(a). In order to protect the heaters from mechanical bombardment which occurs when an ink bubble collapses on the heater surface, the heaters are covered by metal shields which were defined by using a sputtering and a lift-off process. Next were the steps for the formation of the polyimide manifold. A $22 \mu \mathrm{m}$ thick photoresist (AZ4620) was first spun and patterned to form the ink cavities, fluid channels and front-end ink reservoirs. In order to define closely-spaced cavities and microchannels, which are typically $22 \mu \mathrm{m}$ high and have a minimum separation of $5 \mu \mathrm{m}$, contrast enhancement material (CEM) [7] was used in the lithographic process. The cavities and reservoirs were formed after the final sacrificial layer removal, as shown in Fig. 2(d). Before spinning coating of polyimide, the sacrificial photoresist was encapsulated with a thin film which was deposited at low temperature. This film separates the sacrificial photoresist from the polyimide to prevent the solvent in the polyimide from mixing with the photoresist.

The next step was the deposition and patterning of polyimide. A photo-sensitive polyimide and a CEM were spin coated and patterned to define the nozzle structure. The application of CEM is important in this processing step because it significantly enhances the accuracy of nozzle definition. The separation film in the nozzle areas were then selectively dislodged using a dry etch, and the sacrificial photoresist was removed using a wet etch in acetone. The polyimide manifold was then cured. Finally, each nozzle head was separated using a dicing saw.

Figure 3 shows the top view photograph of a 16 nozzle array. The circular part represents the polyimide manifold, in which the nozzles are aligned in the center with two semicircular ink supply reservoirs. The thin film electrical interconnect leads and their bonding pads can be clearly seen. Figure 4 is a close-up of this nozzle array. The diameter of each nozzle is $28 \mu \mathrm{m}$ while the nozzle-to-nozzle separation is $10 \mu \mathrm{m}$, resulting in a 668 dots per inch resolution.

\section{TESTING RESULTS}

The polyimide manifold is transparent. After the cavity is filled with ink, the movement of the ink in the manifold can be clearly seen during the printing process. The temperature coefficient of resistance (TCR) of the micro heater is about $1100 \mathrm{ppm} /{ }^{\circ} \mathrm{C}$, providing a $0.3^{\circ} \mathrm{C}$ resolution when working as a 
temperature sensor. A series of life tests and ink refill efficiency measurements are now being undertaken to assess the performance of this nozzle head.

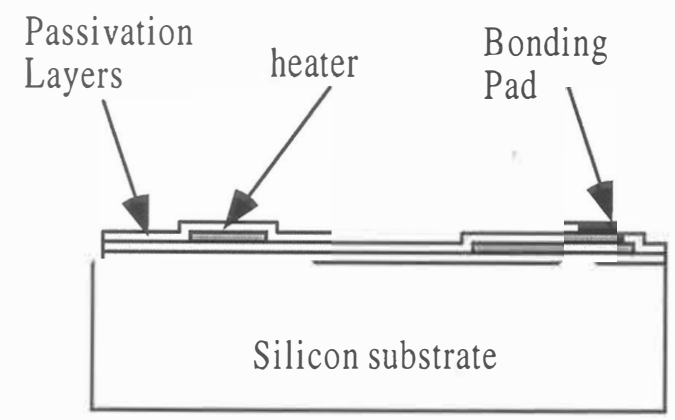

(a) Formation of a micro heater array.

\section{Photoresist}

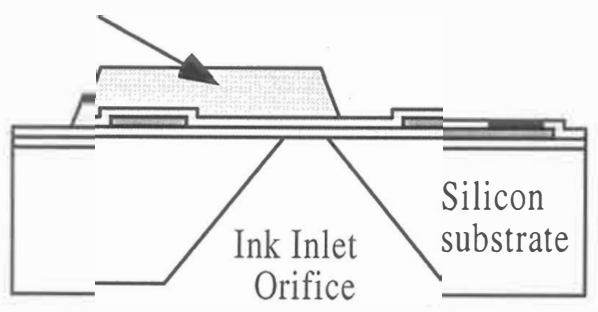

(b) Etch via holes for ink supply, formation of a thick photoresist sacrificial layer.

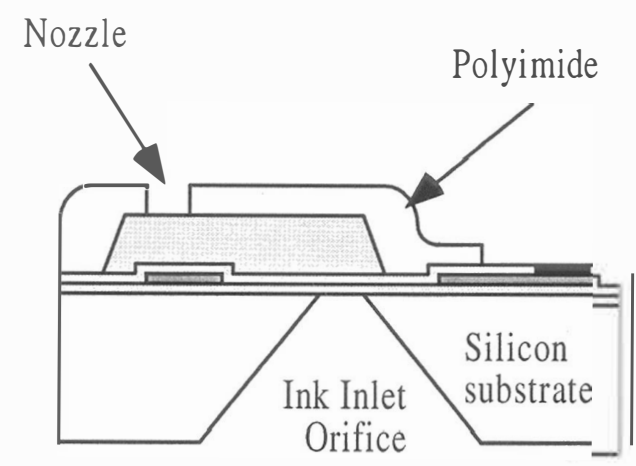

(c) Spin coating and patterning of polyimide

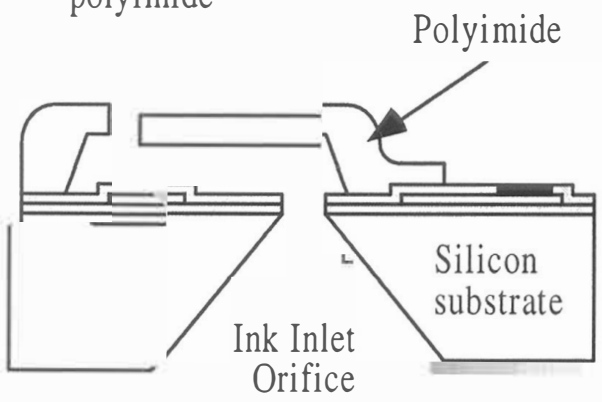

(d) Removal of sacrificial photoresist, completely opening of via holes.

Figure 2: Process sequence for the fabrication of a monolithic polyimide nozzle array for inkjet printing.

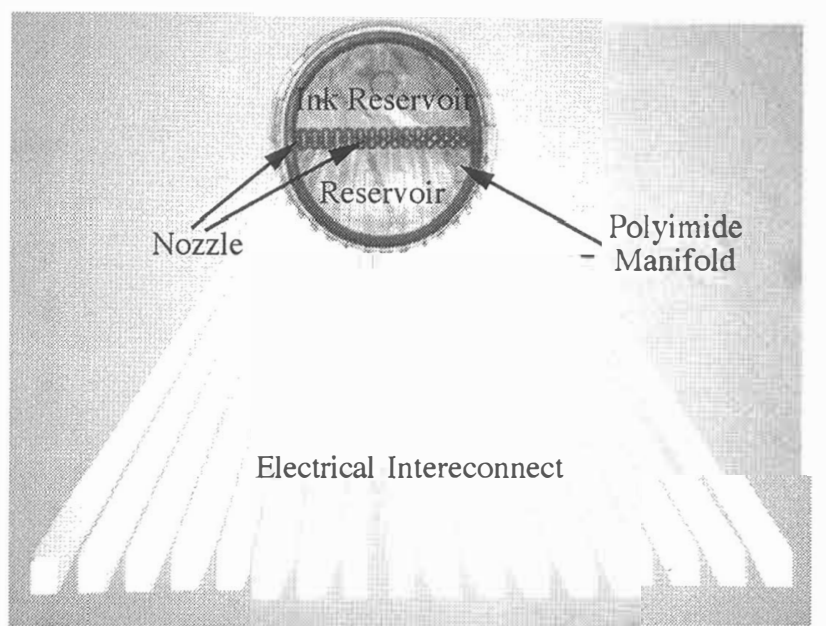

Figure 3: Top view of a 16 nozzle array.

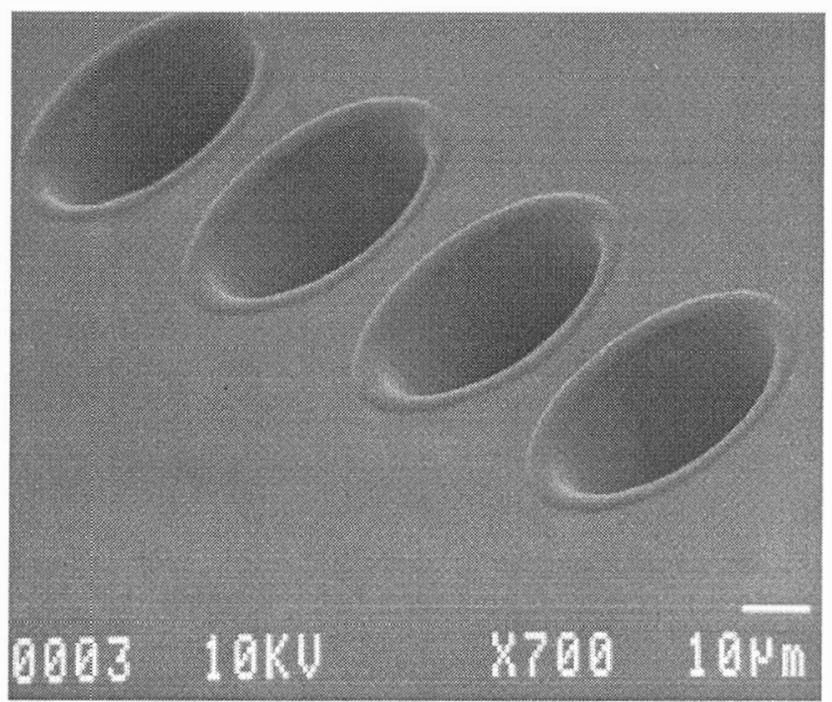

Figure 4: SEM photograph of a polyimide nozzle array defined using a direct photolithographic process on a photosensitive polyimide.

\section{CONCLUSIONS}

A fully monolithic polyimide nozzle array for inkjet printing has been developed. This print head was realized by molding polyimide nozzles on top of a silicon wafer with micro actuators. On this thermal inkjet print head, closely spaced nozzles and $22 \mu \mathrm{m}$ high micro cavities/channels are formed using a direct photolithographic process on a photosensitive polyimide and a sacrificial etch on a thick photoresist. The formation of these polyimide microfluidic structures is a low temperature $\left(<300^{\circ} \mathrm{C}\right)$ process, allowing integration of polyimide microfluidic elements with thin-film electrical interconnects, micro heaters, CMOS circuitry, and/or other micro-electro-mechanical components on silicon for fluid manipulation. The integration of these electrical and fluidic elements into a microfluidic system allows for the fabrication of a low-cost monolithic ink jet printhead.

Nozzle arrays with densities ranging from 300 dots per inch (dpi) to 800 dpi have been designed and fabricated. Different geometrical configurations of ink cavity-reservoir connection have also been designed and are currently being tested for optimizing the 
ink supply efficiency. The key step of this printhead fabrication process is the protection of the structural polyimide from mixing with the sacrificial photoresist underneath. Various separation materials have been tried and optimal candidates have been identified for inkjet applications. This novel fabrication approach improves upon existing technology which uses bonding to assemble parts into a printhead by using a simpler and more costeffective fabrication process that produces improved resolution. The technology can also be combined with an implementation of multi-polysilicon-layer surface micromachined structures to realize various microfluidic systems for chemical analysis and for biomedical applications.

\section{REFERENCES}

1. G. L. Siewell, W. R. Boucher, and P. H. McClelland, "The ThinkJet Orifice Plate: A Part with Many Function," HP Journal, pp. 33-37, May 1985.

2. J. Lee, H. D. Lee, H. J. Lee, J. Yoon, K. Han, J. Kim, C. Kim, and C. Han, "A Monolithic Thermal Inkjet Printhead Utilizing Electrochemical Etching and Two-Step Electroplating Techniques," 1995 IEDM, pp. 601-604.

3. Y. Lin, P. Hesketh, and J. Boyd, "Characteristics of a Polyimide Microvalve," 1996 IEEE Solid-State Sensor and Actuator Workshop, Hilton Head, S.C., pp. 113-116.

4. J. Chen and K. D. Wise, "A Silicon Probe with Integrated Microheater for Thermal Marking and Monitoring of Neural Tissue," IEEE Trans. Biomd. Eng., vol. 44, pp. 770-774, August 1997.

5. Frank M. White., "Fluid Mechanics," 2nd Ed. McGraw-Hill, 1986.

6. S. Pond, "Drop-On-Demand Ink Jet Transducer Effective-ness," IS\&T's Tenth International Congress on Advances in Non-Impact Printing Technologies, 1994, pp. 414-417.

7. H. Miyajima and M. Mehregany, "High-Aspect-Ratio Photolithography for MEMS Applications," Journal of Microelectromechanical Systems, vol. 4, pp.220-229, Dec. 1995. 